\title{
THE GENERAL THEORY OF A CLASS OF LINEAR PARTIAL $q$-DIFFERENCE EQUATIONS*
}

BY

\section{RAYMOND ADAMS}

INTRODUCTION

During the past fifteen years a great deal of work has been done in building up a general theory of the linear difference equation in one independent variable, or linear ordinary difference equation.t No study seems to have been made, however, of the linear difference equation in more than one independent variable, or linear partial difference equation.

Essentially two distinct types of ordinary difference equations have been subjected to investigation: (i) that usually spoken of simply as the linear difference equation, $\ddagger$ and (ii) that which has received the name of linear $q$-difference equation. $\S$ These suggest three types of linear partial difference equations in two independent variables, which (if we confine our attention to homogeneous equations) may be written as follows:

$$
\begin{array}{r}
\sum_{\mu=0}^{m} \sum_{\nu=0}^{n} a_{\mu \nu}(x, y) g(x+m-\mu, y+n-\nu)=0 \\
\sum_{\mu=0}^{m} \sum_{\nu=0}^{n} a_{\mu \nu}(x, y) g\left(q^{m-\mu} x, r^{n-\nu} y\right)=0 \\
\sum_{\mu=0}^{m} \sum_{\nu=0}^{n} a_{\mu \nu}(x, y) g\left(x+m-\mu, r^{n-\nu} y\right)=0
\end{array}
$$

in which $q$ and $r$ are constants, real or complex and different from zero; the $a_{\mu \nu}(x, y)$ are known analytic functions of $x$ and $y$; and $g(x, y)$ is the

* Presented to the Society, December 28, 1923.

† For a comprehensive survey of what has been done, see Nörlund, Neuere Untersuchungen über Differenzengleichungen, Encyklopädie der mathematischen Wissenschaften, vol. $\Pi_{3}$, No. 6, Leipzig, Teubner, 1923, pp. 675-721.

$\ddagger$ Cf. Birkhoff, General theory of linear difference equations, these Transactions, vol. 12 (1911), pp. 243-284.

§ Cf. Carmichael, The general theory of linear q-difference equations, A m eric an Journal of Mathematics, vol. 34 (1912), pp. 147-168. 
function to be determined. To the equations $(A),(B)$, and $(C)$ we give respectively the names linear partial pure difference equation, linear partial $q$-difference equation, and linear partial difference equation of the intermediate type. If in $(A),(B)$, and $(C) \mu$ be set equal to $\nu$ and $m$ equal to $n$, the following important classes of these partial difference equations are obtained:

$$
\begin{aligned}
\sum_{\nu=0}^{n} a_{\nu}(x, y) g(x+n-\nu, y+n-\nu) & =0, \\
\sum_{\nu=0}^{n} a_{\nu}(x, y) g\left(q^{n-\nu} x, r^{n-\nu} y\right) & =0, \\
\sum_{\nu=0}^{n} a_{\nu}(x, y) g\left(x+n-\nu, r^{n-\nu} y\right) & =0 .
\end{aligned}
$$

It is the purpose of the present paper to develop a general theory of equations of the class $\left(B^{\prime}\right)$; equations of classes $\left(A^{\prime}\right)$ and $\left(C^{\prime}\right)$ will be treated later. For the most part the methods used are along the lines of those employed by Birkhoff* in his work on ordinary difference equations. In $\S 1$, formal series solutions are found; $\S 2$ is devoted to proving the convergence of these series. In $\S 3$ are considered certain cases to which the methods of $\S \S 1,2$ do not apply. The object of $\S 4$ is to indicate that when $|q|=|r|=1$, the equation in general has no analytic solution. The periodic functions which may be defined in terms of two solutions of an equation of this type are discussed in $\S 5$. In $\S 6$ a theorem is proved which is in part an inverse of the results obtained in the earlier work.

It should be observed that the chief results of the paper are immediately extensible to the case of an equation of the class $\left(B^{\prime}\right)$ in $N$ independent variables; i. e.,

$$
\sum_{\nu=0}^{n} a_{\nu}(x, y, \ldots, w) g\left(q^{n-\nu} x, r^{n-\nu} y, \ldots, s^{n-\nu} w\right)=0 .
$$

The author wishes to express his sincere thanks to Professor George D. Birkhoff, whose valuable suggestions have been of aid in the preparation of this paper.

\section{The Formal Series SOLutions}

The equation of the nth order of the class $\left(B^{\prime}\right)$ will be defined as

$$
\sum_{\nu=0}^{n} p_{\nu}(x, y) g\left(q^{n-\nu} x, r^{n-\nu} y\right)=0,
$$

\footnotetext{
${ }^{*}$ Loc. cit.
} 
in which $q$ and $r$ are real or complex constants not zero; the $p_{\nu}(x, y)$ are arbitrary known functions of the complex variables $x$ and $y$ and $p_{0}(x, y)$ 末 0 ; and $g(x, y)$ is the function to be determined. It is important to observe first of all that

Every equation of the nth order of the class $\left(B^{\prime}\right)$ can be solved by determining the solutions of an associated system of $n$ equations of the first order of the class $\left(B^{\prime}\right)$.

In fact if we define

$$
g_{i}(x, y)=g\left(q^{i-1} x, r^{i-1} y\right) \quad(i=1,2, \ldots, n),
$$

the equation (1) is seen to be equivalent to the system

$$
\begin{array}{rr}
g_{i}(q x, r y)=g_{i+1}(x, y) & (i=1,2, \ldots, n-1), \\
g_{n}(q x, r y)=-\frac{p_{n}(x, y)}{p_{0}(x, y)} g_{1}(x, y)-\frac{p_{n-1}(x, y)}{p_{0}(x, y)} g_{2}(x, y)-\cdots \\
\ldots-\frac{p_{1}(x, y)}{p_{0}(x, y)} g_{n}(x, y) .
\end{array}
$$

We propose to show that an equation of the type (1) can in general be solved when the coefficients $p_{\nu}(x, y)$ are polynomials in $x$ and $y .^{*}$ In that case the coefficients in the equivalent system (2), with which we shall deal, will be rational functions. It is unnecessary, however, for us to develop a theory for the solution of a system of equations with rational coefficients, for

The solution of a system of $n$ equations of the first order of the class $\left(B^{\prime}\right)$ with rational coefficients can in general be made to depend upon the solution of a second such system with polynomial coefficients.

Proof. Let

$$
g_{i}(q x, r y)=\sum_{j=1}^{n} r_{i j}(x, y) g_{j}(x, y) \quad(i=1,2, \ldots, n),
$$

or in the matrix notation

$$
G(q x, r y)=R(x, y) G(x, y)
$$

be the given system. With no loss of generality it may and will be assumed that each rational function $r_{i j}(x, y)$ is expressed as the quotient of

* The problem is clearly the same as if the coefficients were taken to be rational functions. 
relatively prime polynomials. If $d(x, y)$ is the least common denominator of the functions $r_{i j}(x, y)$, we have

$$
R(x, y)=\frac{1}{d(x, y)} P(x, y),
$$

where $P(x, y)$ is a matrix of polynomials. It will appear in the course of our later work (cf. $\S \S 2,3$ ) that, $d(x, y)$ being a polynomial, solutions can in general be found for the equation

$$
g(q x, r y)=d(x, y) g(x, y) .
$$

Let one such solution be denoted by $\Phi(x, y)$ and set

$$
G(x, y)=\frac{1}{\Phi(x, y)} \bar{G}(x, y)
$$

then equation (3) becomes directly

$$
\bar{G}(q x, r y)=P(x, y) \bar{G}(x, y) .
$$

It is the chief purpose of the present paper to find analytic solutions of the system

$$
g_{i}(q x, r y)=\sum_{j=1}^{n} p_{i j}(x, y) g_{j}(x, y) \quad(i=1,2, \ldots, n),
$$

where $p_{i j}(x, y)$ is a polynomial

$$
p_{i j}(x, y)=p_{i j}+p_{i j 10} x+p_{i j 01} y+\cdots+p_{i j l m} x^{l} y^{m} .
$$

In the matrix notation the system (4) is written

$$
G(q x, r y)=P(x, y) G(x, y) \text {. }
$$

Iteration of this equation suggests as symbolic solutions the two infinite products of matrices

$$
\begin{gathered}
P\left(\frac{x}{q}, \frac{y}{r}\right) P\left(\frac{x}{q^{2}}, \frac{y}{r^{2}}\right) P\left(\frac{x}{q^{3}}, \frac{y}{r^{3}}\right) \cdots \\
P^{-1}(x, y) P^{-1}(q x, r y) P^{-1}\left(q^{2} x, r^{2} y\right) \cdots
\end{gathered}
$$

Certain modifications of these will play an important part in our work. 
Among all the polynomials $p_{i j}(x, y)$ there will be one (or more) whose degree in $x$ is the greatest; let that degree be $\mu$ : similarly there will be one (or more) whose degree in $y$ is the greatest; let that degree be $\nu$. Then the following determinantal equations will be called the characteristic equations:

$$
\begin{aligned}
& \text { for }(0,0) \text {, } \\
& \left|p_{i j}-\delta_{i j} \sigma\right|=0 \\
& \text { for }(\infty, \infty) \text {, } \\
& \left|p_{i j \mu \nu}-\delta_{i j} \sigma^{\prime}\right|=0 \\
& \text { for }(0, \infty) \text {, } \\
& \left|p_{i j 0 \nu}-\delta_{i j} \sigma^{\prime \prime}\right|=0 \\
& \text { for }(\infty, 0) \text {, } \\
& \left|p_{i j \mu 0}-\delta_{i j} \sigma^{\prime \prime \prime}\right|=0 \\
& \left(\delta_{i j}=\left\{\begin{array}{l}
0, i \neq j \\
1, i=j
\end{array}\right) .\right.
\end{aligned}
$$

The roots of these equations will be designated respectively by $\sigma_{i}, \sigma_{i}^{\prime}, \sigma_{i}^{\prime \prime}$, and $\sigma_{i}^{\prime \prime}(i=1,2, \ldots, n)$.

Our attention will at first be confined to the case in which the roots $\sigma_{i}, \sigma_{i}^{\prime}, \sigma_{i}^{\prime \prime}$, and $\sigma_{i}^{\prime \prime \prime}$ are all different from zero.* We shall make the further restriction that

$$
\begin{array}{ll}
\frac{\sigma_{i}}{\sigma_{j}} \neq q^{\beta} r^{\gamma}, & \frac{\sigma_{i}^{\prime}}{\sigma_{j}^{\prime}} \neq q^{\beta} r^{\gamma}, \\
\frac{\sigma_{i}^{\prime \prime}}{\sigma_{j}^{\prime \prime}} \neq q^{\beta} r^{\gamma}, & \frac{\sigma_{i}^{\prime \prime \prime}}{\sigma_{j}^{\prime \prime \prime}} \neq q^{\beta} r^{\gamma}
\end{array}
$$

for any $\beta$ and $\gamma$ which are positive integers or zero (but not both zero). Under these conditions and if we have $|q|,|r| \neq 1$, and if the roots of each characteristic equation are distinct, it is found by direct substitution and reckoning of the coefficients that the system (4) is satisfied formally (a) by each of $n$ sets of functions

$$
\begin{gathered}
s_{1 j}(x, y)=x^{\rho_{j}\left[s_{1 j}+s_{1 j 10} x+s_{1 j 01} y+\cdots\right]} \\
. . . . . . . . . . . . \\
s_{n j}(x, y)=x^{\rho_{j}\left[s_{n j}+s_{n j 10} x+s_{n j 01} y+\ldots\right]} \\
(j=1,2, \ldots, n),
\end{gathered}
$$$$
\text { (6) }
$$

*A method by which solutions can frequently (in fact "in general" for the case of a single equation) be obtained when this condition does not hold will be given in $\S 3$. 
where the $\varrho_{j}$ are determined by the relations

$$
q^{\rho_{j}}=\sigma_{j} \text { or } \varrho_{j}=\log _{q} \sigma_{j}=\frac{\log \sigma_{j}}{\log q}
$$

(b) by each of $n$ sets of functions

$$
s_{1 j}^{\prime}(x, y)=q^{\mu(t)-t) / 2} r^{\nu\left(\tau^{s}-\tau\right) / 2} x^{\rho_{j}^{\prime}}\left[s_{1 j}^{\prime}+\frac{s_{1 j 10}^{\prime}}{x}+\frac{s_{1 j 01}^{\prime}}{y}+\cdots\right],
$$

$$
s_{n j}^{\prime}(x, y)=q^{\mu\left(t^{2}-t\right) / 2} r^{\nu\left(\tau^{2}-\tau\right) / 2} x^{\rho_{j}^{\prime}}\left[s_{n j}^{\prime}+\frac{s_{n j 10}^{\prime}}{x}+\frac{s_{n j 01}^{\prime}}{y}+\cdots\right]
$$

in which

$$
(j=1,2, \ldots, n),
$$

$$
t=\frac{\log x}{\log q}, \quad \tau=\frac{\log y}{\log r},
$$

and the $\varrho_{j}^{\prime}$ are determined as

$$
\varrho_{j}^{\prime}=\frac{\log \sigma_{j}^{\prime}}{\log q}
$$

(c) by each of $n$ sets of functions

(8)

$$
s_{1 j}^{\prime \prime}(x, y)=r^{\nu\left(\tau^{*}-\tau\right) / 2} x^{\rho_{j}^{\prime \prime}}\left[s_{1 j}^{\prime \prime}+s_{1 j 10}^{\prime \prime} x+\frac{s_{j j 01}^{\prime \prime}}{y}+\cdots\right],
$$

$$
\begin{gathered}
s_{n j}^{\prime \prime}(x, y)=r^{\nu\left(\tau^{2}-\tau\right) / 2} x^{\rho_{j}^{\prime \prime}}\left[s_{n j}^{\prime \prime}+s_{n j 10}^{\prime \prime} x+\frac{s_{n j 01}^{\prime \prime}}{y}+\cdots\right] \\
(j=1,2, \ldots, n),
\end{gathered}
$$

where

$$
\varrho_{j}^{\prime \prime}=\frac{\log \sigma_{j}^{\prime \prime}}{\log q}
$$

and (d) by each of $n$ sets of functions

$$
s_{1 j}^{\prime \prime \prime}(x, y)=q^{\mu\left(t^{2}-t\right) / 2} x^{\rho_{j}^{\prime \prime \prime}}\left[s_{1 j}^{\prime \prime \prime}+\frac{s_{1 j 10}^{\prime \prime \prime}}{x}+s_{1 j 01}^{\prime \prime \prime} y+\cdots\right],
$$

(9)

$$
\begin{gathered}
s_{n j}^{\prime \prime \prime}(x, y)=q^{\mu\left(t^{2}-t\right) / 2} x^{\rho_{j}^{\prime \prime \prime}}\left[s_{n j}^{\prime \prime \prime}+\frac{s_{n j 10}^{\prime \prime \prime}}{x}+s_{n j 01}^{\prime \prime \prime} y+\cdots\right] \\
(j=1,2, \ldots, n),
\end{gathered}
$$


in which

$$
\varrho_{j}^{\prime \prime \prime}=\frac{\log \sigma_{j}^{\prime \prime \prime}}{\log q}
$$

Furthermore the sets of functions (6), and similarly (7), (8), and (9), are linearly independent. Throughout our work those determinations of $\log q$ and $\log r$ will be taken in which the coefficient of $\sqrt{-1}$ is positive or zero and less than $2 \pi$. $^{*}$

We shall not assume, however, that the roots of each characteristic equation are distinct, but we shall make the hypothesis that the formal series solutions (6), (7), (8), and (9) do exist and that they are such that the following determinantal inequalities hold:

$$
\begin{aligned}
d=\left|s_{i j}\right| \neq 0, & d^{\prime}=\left|s_{i j}^{\prime}\right| \neq 0, \\
d^{\prime \prime}=\left|s_{i j}^{\prime \prime}\right| \neq 0, & d^{\prime \prime \prime}=\left|s_{i j}^{\prime \prime}\right| \neq 0 .
\end{aligned}
$$

The $n$ sets of formal solutions (6) are then linearly independent and constitute a matrix $S(x, y)$ which is a formal matrix solution of the equation (5). The same statement may be made for the solutions (7), (8), and $(9)$; let the respective matrices be denoted by $S^{\prime}(x, y), S^{\prime \prime}(x, y)$, and $S^{\prime \prime \prime}(x, y)$. It will be demonstrated $(a)$ that if $|q|$ and $|r|$ are both greater or both less than unity, the series in the matrix $S(x, y)\left[S^{\prime}(x, y)\right]$ converge uniformly and absolutely in the neighborhood of any placet $(x, y)$ for which $x$ and $y$ respectively lie inside [outside] certain associated circles $r_{1}, r_{2}\left[R_{1}, R_{2}\right]$ about the origins in their respective planes; and $(b)$ that if one of the quantities $|q|$ and $|r|$ is greater and the other less than unity, the series in the matrix $S^{\prime \prime}(x, y)\left[S^{\prime \prime \prime}(x, y)\right]$ converge uniformly and absolutely in the neighborhood of any place $(x, y)$ for which $x$ and $y$ lie respectively inside [outside] and outside [inside] certain associated circles $r_{1}, R_{2}\left[R_{1}, r_{2}\right]$ about the origins in their respective planes.

The most general analytic matrix solution of (5) is given by

$$
H(x, y)=G(x, y) A(x, y)
$$

* It is to be noted that the power of $x$ occurring immediately before the bracketed series in each set of formal series solutions is not a uniquely determined factor; in place of $x^{\rho_{j}}$ in (6), for example, we may equally well use $y^{\rho_{j}}$ or $(x y)^{\rho_{j}}$ or, in fact, any function $f(x, y)$ such that $f(q x, r y)=c f(x, y)$, where $c$ is a constant.

$\dagger$ We shall find it convenient to think of the complex variables $x$ and $y$ as represented by points in two distinct planes. In conformity with the usual convention a pair of values of $x$ and $y$ will be spoken of as the place $(x, y)$. 
where $G(x, y)$ is any particular analytic matrix solution, and $A(x, y)$ is any arbitrary matrix of analytic functions which possesses the multiplicative period system* $(q, r)$ and whose determinant $|A(x, y)|$ is not identically zero.

\section{EXISTENCE THEOREMS}

Of the four cases $|q| \gtrless 1,|r| \gtrless 1$ which are to be considered in this section, that in which both absolute values are greater than unity is wholly typical. We shall therefore confine ourselves in giving detailed discussion to

\section{The Case of $|q|,|r|>1$.}

It has been observed that the matrix $S(x, y)$ is a formal matrix solution of equation (5); that is, that

$$
S(q x, r y)=P(x, y) S(x, y) .
$$

Now the determinant $|S(x, y)|$ may be written

$$
x^{\rho_{1}+\rho_{2}+\cdots+\rho_{n}}\left[d+d_{10} x+d_{01} y+\cdots\right] .
$$

The element in the $i$ th row and $j$ th column of the inverse matrix is the quotient of the cofactor of the element in the $j$ th row and $i$ th column of this determinant by the determinant itself, and is therefore given by a series

$$
\bar{s}_{i j}(x, y)=x^{-\rho_{i}}\left[\bar{s}_{i j}+\bar{s}_{i j 10} x+\bar{s}_{i j 01} y+\cdots\right]
$$

Let $T(x, y)$ denote the matrix obtained by breaking off the elements of $S(x, y)$ so as to retain in the series only the terms of degree less than or equal to $k-1$, or more generally by replacing $s_{i j}(x, y)$ by $t_{i j}(x, y)$, where the series in the latter is convergent for $(x, y)$ in the vicinity of the place $(0,0)$ and has the same terms as the series in $s_{i j}(x, y)$ up to and including those of degree $k-1$. We may then define a matrix $Q(x, y)$ by the relation

$$
T(q x, r y)=Q(x, y) T(x, y) .
$$

* The matrix $A(x, y)$ shall be said to possess the multiplicative period system $(q, r)$ if $A(q x, r y)=A(x, y)$. 
The matrix $Q(x, y)$ is a matrix of functions $q_{i j}(x, y)$ each of which has an expansion in powers of $x$ and $y$ that agrees precisely with $p_{i j}(x, y)$ up to and including terms of degree $k-1$. This follows upon comparing

$$
Q(x, y)=T(q x, r y) T^{-1}(x, y) \text { and } P(x, y)=S(q x, r y) S^{-1}(x, y)
$$

for $T(q x, r y)$ is the same as $S(q x, r y)$ up to and including terms of degree $k-1$, and similarly for $T^{-1}(x, y)$ and $S^{-1}(x, y)$, because $\bar{t}_{i j}(x, y)$ of $T^{-1}(x, y)$ is given by (11) up to and including terms of degree $k-1$. We therefore have

$$
P(x, y)=Q(x, y)+M(x, y),
$$

where $M(x, y)$ is a matrix of power series in $x$ and $y$ whose lowest degree terms are of degree $k$ or higher and which converge in the vicinity of the place $(0,0)$. Then $N(x, y)$, defined by

$$
M(x, y)=Q(x, y) N(x, y)
$$

is a matrix of power series of the same type as those in $M(x, y)$. Hence

$$
P(x, y)=Q(x, y)[I+N(x, y)]
$$

in which $I$ is the unit matrix and $N(x, y)$ is a matrix of power series each of which contains no term of degree less than $k$ and which converges in the vicinity of $(0,0)$.

The0Rem I, A. Form the product of matrices

$$
P_{m}(x, y)=P\left(\frac{x}{q}, \frac{y}{r}\right) P\left(\frac{x}{q^{2}}, \frac{y}{r^{2}}\right) \ldots P\left(\frac{x}{q^{m}}, \frac{y}{r^{m}}\right) T\left(\frac{x}{q^{m}}, \frac{y}{r^{m}}\right) .
$$

Each element of $P_{m}(x, y)$ converges, for $k$ sufficiently large, to a definite limit function $u_{i j}(x, y)$, independent of $k$, as $m$ becomes infinite. This function is analytic throughout the entire finite $x$ - and $y$-planes except perhaps at $x=0$, and in this region is identical with the corresponding element $s_{i j}(x, y)$ of $S(x, y)$.

Proof. We may write

$$
P_{m}(x, y)=T(x, y) \bar{P}_{m}(x, y),
$$


where

$$
\begin{aligned}
\bar{P}_{m}(x, y)=\left[T^{-1}(x, y) P\left(\frac{x}{q}, \frac{y}{r}\right) T\left(\frac{x}{q}, \frac{y}{r}\right)\right] \\
\cdot\left[T^{-1}\left(\frac{x}{q}, \frac{y}{r}\right) P\left(\frac{x}{q^{2}}, \frac{y}{r^{2}}\right) T\left(\frac{x}{q^{2}}, \frac{y}{r^{2}}\right)\right] \ldots \\
\ldots\left[T^{-1}\left(\frac{x}{q^{m-1}}, \frac{y}{r^{m-1}}\right) P\left(\frac{x}{q^{m}}, \frac{y}{r^{m}}\right) T\left(\frac{x}{q^{m}}, \frac{y}{r^{m}}\right)\right] .
\end{aligned}
$$

The elements of $T(x, y)$ are polynomials or series convergent in the vicinity of $(0,0)$ (multiplied by a power of $x$ ). In order to show that the elements of $P_{m}(x, y)$ converge, it will be sufficient to prove that those of $\bar{P}_{m}(x, y) \mathrm{do}$. We proceed to a proof of this fact.

The matrix $\bar{P}_{m}(x, y)$ is the product of matrices of the type

$$
T^{-1}(q x, r y) P(x, y) T(x, y)=I+T^{-1}(x, y) N(x, y) T(x, y)
$$

The second term is a matrix

$$
\left(x^{\rho_{j}-\rho_{i}} \lambda_{i j}(x, y)\right)=\left(\theta_{i j}(x, y)\right)=\Theta(x, y)
$$

where the $\lambda_{i j}(x, y)$ stand for power series in $x$ and $y$ whose lowest degree terms are of degree $k$ or higher and which converge in the neighborhood of $(0,0)$.

The matrix $\bar{P}_{m}(x, y)$ may now be written

$$
\begin{aligned}
{\left[I+\Theta\left(\frac{x}{q}, \frac{y}{r}\right)\right]\left[I+\Theta\left(\frac{x}{q^{2}}, \frac{y}{r^{2}}\right)\right] \ldots\left[I+\Theta\left(\frac{x}{q^{m}}, \frac{y}{r^{m}}\right)\right] } \\
=I+\sum_{k_{1}=1}^{m} \Theta\left(\frac{x}{q^{k_{1}}}, \frac{y}{r^{k_{1}}}\right)+\sum_{k_{1}=1}^{m-1} \sum_{k_{3}=k_{1}+1}^{m} \Theta\left(\frac{x}{q^{k_{1}}}, \frac{y}{r^{k_{1}}}\right) \Theta\left(\frac{x}{q^{k_{2}}}, \frac{y}{r^{k_{2}}}\right)+\cdots
\end{aligned}
$$

The $i$ th element in the $j$ th column $(i, j=1,2, \ldots, n)$ is

$$
\begin{aligned}
\bar{p}_{m ; i j}(x, y)=\delta_{i j} & +\sum_{k_{1}=1}^{m} \theta_{i j}\left(\frac{x}{q^{k_{1}}}, \frac{y}{r^{k_{1}}}\right) \\
& +\sum_{k_{1}=1}^{m-1} \sum_{k_{2}=k_{1}+1}^{m}\left[\sum_{\tau=1}^{n} \theta_{i \tau}\left(\frac{x}{q^{k_{1}}}, \frac{y}{r^{k_{1}}}\right) \theta_{\tau j}\left(\frac{x}{q^{k_{2}}}, \frac{y}{r^{k_{2}}}\right)\right]+\cdots,
\end{aligned}
$$

$\delta_{i j}$ denoting the element in the $i$ th row and $j$ th column of the unit matrix. 
Let it next be noted that the product of two (and hence of $N$ ) matrices of the type of $\Theta(x, y)$ which differ only in the series $\lambda$ is another matrix of the same type. For, if the two factor matrices are $\Theta(x, y)$ and $\Theta^{\prime}(x, y)$ and their product is $\Theta^{\prime \prime}(x, y)$, we have (denoting by [ג] a series of the type $\lambda$ )

$$
\begin{aligned}
\theta_{i j}^{\prime \prime} & =\theta_{i 1} \theta_{1 j}^{\prime}+\theta_{i 2} \theta_{2 j}^{\prime}+\cdots+\theta_{i n} \theta_{n j}^{\prime} \\
& =x^{\rho_{1}-\rho_{i}} x^{\rho_{j}-\rho_{1}}[\lambda][\lambda]+x^{\rho_{2}-\rho_{i}} x^{\rho_{j}-\rho_{2}}[\lambda][\lambda]+\cdots+x^{\rho_{n}-\rho_{i}} x^{\rho_{j}-\rho_{n}}[\lambda][\lambda] \\
& =x^{\rho_{j}-\rho_{i}}[\lambda] .
\end{aligned}
$$

This remark makes it clear that we have

$$
x^{\rho_{i}-\rho_{j}} \bar{p}_{m ; i j}-\delta_{i j}=\sum_{k_{1}=1}^{m} \lambda_{i j}\left(\frac{x}{q^{k_{1}}}, \frac{y}{r^{k_{1}}}\right) \frac{1}{q^{\left(\rho_{j}-\rho_{i}\right) k_{1}}}
$$

$$
+\sum_{k_{1}=1}^{m-1} \sum_{k_{2}=k_{1}+1}^{m}\left[\sum_{\tau=1}^{n} \lambda_{i \tau}\left(\frac{x}{q^{k_{1}}}, \frac{y}{r^{k_{1}}}\right) \lambda_{\tau j}\left(\frac{x}{q^{k_{2}}}, \frac{y}{r^{k_{2}}}\right) \frac{1}{q^{\left.\left(\rho_{\tau}-\rho_{i}\right) k_{1}+\rho_{j}-\rho_{\tau}\right) k_{2}}}\right]+\cdots
$$

If $p$ is equal to the smaller of the two quantities $|q|$ and $|r|$ and if $x$ and $y$ are inside certain associated circles $r_{1}, r_{2}$ about the origins in their respective planes, then the typical element in the $l$ th term of the right-hand member of (13) is less in absolute value than

$$
\frac{M^{l}}{p^{\left(k_{1}+k_{2}+\cdots+k_{\imath}\right) k}\left|q^{\left(\rho_{\tau}-\rho_{i}\right) k_{1}+\left(\rho_{\sigma}-\rho_{\tau}\right) k_{2}+\cdots+\left(\rho_{j}-\rho_{\theta}\right) k_{l}}\right|}
$$

because when $(x, y)$ is in this region all the functions $\lambda_{i j}(x, y)$ are less than some constant $M$. Furthermore if $s$ is a positive integer assigned arbitrarily, we can take $k$ so large that the inequality

$$
p^{k}\left|q^{\rho_{\alpha}-\rho_{\beta}}\right|>p^{s}
$$

will hold for $\alpha, \beta=1,2, \ldots, n$. Let any positive integer $s$ be assigned and a suitable $k$ be found; it follows that the series (13) will then be less term by term in absolute value than the series

$$
\sum_{k_{1}=1}^{m} \frac{M}{p^{k_{1} s}}+\sum_{k_{1}=1}^{m-1} \sum_{k_{2}=k_{1}+1}^{m} \frac{n M^{2}}{p^{\left(k_{1}+k_{s}\right) s}}+\cdots
$$


As $m$ is allowed to increase without limit this series approaches the valu

The ratio

$$
\frac{1}{n}\left\{\left[\left(1+\frac{n M}{p^{s}}\right)\left(1+\frac{n M}{p^{2 s}}\right) \cdots\right]-1\right\}
$$

$$
\frac{1}{p^{n s}} \div \frac{1}{p^{(n-1) s}}=\frac{1}{p^{s}}
$$

since $p$ is $>1$ and $s$ is a positive integer, is less than unity. The element in the $i$ th row and $j$ th column of $\bar{P}_{m}(x, y)$, after being multiplied by $x^{\rho_{i}-\rho_{s}}$, therefore converges uniformly and absolutely to a function analycic in the neighborhood of places $(x, y)$ such that $|x|$ is $\left\langle r_{1}\right.$ and $|y|<r_{2}$. Hence each element of $P_{m}(x, y)$ converges in a similar manner in the neighborhood of places $(x, y)$ such that $0 \neq|x|<r_{1},|y|<r_{2}$.

We may write

$$
P_{m}(x, y)=P\left(\frac{x}{q}, \frac{y}{r}\right) P\left(\frac{x}{q^{2}}, \frac{y}{r^{2}}\right) \ldots P\left(\frac{x}{q^{t}}, \frac{y}{r^{t}}\right) P_{m-t}\left(\frac{x}{q^{t}}, \frac{y}{r^{t}}\right)
$$

and apply the argument as above to $P_{m-t}\left(x / q^{t}, y / r^{t}\right)$. It follows, since $|q|$ and $|r|$ are $>1$, that if any place $(x, y)$ be assigned where $x$ and $y$ are in the finite parts of their respective planes and $x \neq 0, t$ can be chosen so large that $\left(x / q^{t}\right)(\neq 0)$ and $y / r^{t}$ will lie within the respective circles $r_{1}$ and $r_{9}$. We conclude that the elements of $P_{m}(x, y)$ converge to functions $u_{i j}(x, y)$ analytic over the entire finite parts of the $x$ - and $y$-planes except at $x=0$.

The functions $u_{i j}(x, y)$ are independent of $k$ (so long as $k$ is sufficiently large), because if $k^{\prime}$ is any second value for $k$ and $T^{\prime}(x, y)$ the matrix corresponding to $T(x, y)$, we may write

$$
\begin{aligned}
& P_{m}^{\prime}(x, y)=T(x, y) {\left[T^{-1}(x, y) P\left(\frac{x}{q}, \frac{y}{r}\right) T\left(\frac{x}{q}, \frac{y}{r}\right)\right] } \\
& \cdot\left[T^{-1}\left(\frac{x}{q}, \frac{y}{r}\right) P\left(\frac{x}{q^{2}}, \frac{y}{r^{2}}\right) T\left(\frac{x}{q^{2}}, \frac{y}{r^{2}}\right)\right] \ldots \\
& \ldots\left[T^{-1}\left(\frac{x}{q^{m-1}}, \frac{y}{r^{m-1}}\right) P\left(\frac{x}{q^{m}}, \frac{y}{r^{m}}\right) T^{\prime}\left(\frac{x}{q^{m}}, \frac{y}{r^{m}}\right)\right] .
\end{aligned}
$$


Then if the elements be expanded in a sum like (12) and $m$ be allowed to become infinite, the resulting multiple series for $u_{i j}(x, y)$ will be term by term identical with that obtained above.

It remains for us to show that if $x$ and $y$ are anywhere in the finite parts of their respective planes and $x \neq 0$, then $u_{i j}(x, y) \equiv s_{i j}(x, y)$. We shall first show this to be the case if $x(\neq 0)$ and $y$ are respectively within the associated circles $r_{1}, r_{2}$. Let $(x, y)$ be any place in this region. For this pair of values the limit function $\bar{u}_{i j}(x, y)$ of the $i$ th element in the $j$ th column of $\bar{P}_{m}(x, y)$ differs from $\delta_{i j}$ by a quantity less in absolute value than

$$
\left|x^{\rho_{j}-\rho_{i}}\right| \frac{1}{n}\left\{\left[\left(1+\frac{n M}{p^{s}}\right)\left(1+\frac{n M}{p^{2 s}}\right) \cdots\right]-1\right\},
$$

which is itself less than

$$
(1 / n)\left|x^{\rho_{3}-\rho_{i}}\right|\left(e^{\sum_{\nu=1}^{\infty} p^{\nu s}}-1\right) .
$$

But

$$
\sum_{\nu=1}^{\infty} \frac{1}{p^{\nu s}}=\frac{1}{p^{s}-1}
$$

since $1 / p^{s}$ is $<1$. Hence we have

$$
\left|\bar{u}_{i j}(x, y)-\delta_{i j}\right|<\frac{\left|x^{\rho_{j}-\rho_{i}}\right|}{n}\left(e^{\frac{n M}{p^{s}-1}}-1\right) .
$$

If $s$ (and with it $k$ ) be allowed to increase without limit, it is clear that $\bar{u}_{i j}(x, y)$ approaches $\delta_{i j}$. Therefore is

$$
u_{i j}(x, y) \equiv s_{i j}(x, y) \quad(i, j=1,2, \ldots, n),
$$

for we have shown $u_{i j}(x, y)$ to be independent of $k$, and as $k$ becomes infinite $t_{i j}(x, y)$ becomes $s_{i j}(x, y)$. Thus the function $u_{i j}(x, y)$ is represented by $s_{i j}(x, y)$ for $0 \neq|x|<r_{1}$ and $|y|<r_{8}$. But the function $u_{i j}(x, y)$ is analytic without singularities of any kind over the entire finite $x$ - and $y$-planes except at $x=0$. Hence the power series in $s_{i j}(x, y)$ converges for all finite values of $x$ and $y$ and represents $x^{-\rho_{j}} u_{i j}(x, y)$ throughout this region; it follows that $u_{i j}(x, y) \equiv s_{i j}(x, y)$ in the region specified.

The proof of the theorem as stated is thus complete. 
We may consider in a similar manner the matrix $S^{\prime}(x, y)$. It has already been noted that this matrix is a second formal solution of the matrix equation (5); that is, that

$$
S^{\prime}(q x, r y)=P(x, y) S(x, y)
$$

which, for our immediate purposes, may be written more conveniently as

$$
S^{\prime}(x, y)=P^{-1}(x, y) S^{\prime}(q x, r y) .
$$

The determinant $\left|S^{\prime}(x, y)\right|$ is of the form

$$
q^{n \mu\left(t^{2}-t\right) / 2} r^{n \nu\left(\tau^{2}-\tau\right) / 2} x^{\rho_{1}^{\prime}+\rho_{2}^{\prime}+\cdots+\rho_{n}^{\prime}}\left[d^{\prime}+\frac{d_{10}^{\prime}}{x}+\frac{d_{01}^{\prime}}{y}+\cdots\right] .
$$

The element in the $i$ th row and $j$ th column of the inverse matrix is consequently seen to be

$$
\bar{s}_{i j}^{\prime}(x, y)=q^{-\mu\left(t^{2}-t\right) / 2} r^{-\nu\left(\tau^{2}-\tau\right) / 2} x^{-\rho_{i}^{\prime}}\left[\bar{s}_{i j}^{\prime}+\frac{\bar{s}_{i j 10}^{\prime}}{x}+\frac{\bar{s}_{i j 01}^{\prime}}{y}+\cdots\right]
$$

If $T^{\prime}(x, y)$ denotes the matrix obtained by replacing $s_{i j}^{\prime}(x, y)$ in $S^{\prime}(x, y)$ by $t_{i j}^{\prime}(x, y)$ where the series in the latter is known to be convergent in the neighborhood of $(\infty, \infty)$ and has the same terms as the series in $s_{i j}^{i}(x, y)$ up to and including those of degree $k-1$ in $1 / x$ and $1 / y$, then we may define a matrix $Q^{\prime-1}(x, y)$ by the relation

$$
T^{\prime}(x, y)=Q^{\prime-1}(x, y) T^{\prime}(q x, r y) .
$$

By comparison of

$Q^{\prime-1}(x, y)=T^{\prime}(x, y) T^{\prime-1}(q x, r y)$ and $P^{-1}(x, y)=S^{\prime}(x, y) S^{\prime-1}(q x, r y)$,

we see that $Q^{\prime-1}(x, y)$ is a matrix of functions $\bar{q}_{i j}^{\prime}(x, y)$ of the form

$$
x^{-\mu} y^{-\nu} \cdot\left[\bar{q}_{i j}^{\prime}+\frac{\bar{q}_{i j 10}^{\prime}}{x}+\frac{\bar{q}_{i j 01}^{\prime}}{y}+\cdots\right],
$$


in which the series has exactly the same terms of degree less than $k$ in $1 / x$ and $1 / y$ as has $x^{\mu} y^{\nu} \bar{p}^{i j}(x, y), \bar{p}^{i j}(x, y)$ standing for the element in the $i$ th row and $j$ th column of the matrix $P^{-1}(x, y)$. It follows that

$$
P^{-1}(x, y)=Q^{\prime-1}(x, y)\left[I+N^{\prime}(x, y)\right]
$$

where the elements of the matrix $N^{\prime}(x, y)$ are power series in $1 / x$ and $1 / y$ which contain no terms of degree lower than $k$ in these variables and which are convergent in the vicinity of $(\infty, \infty)$.

We may now state

TheOREM I, B. Form the product of matrices

$\Pi_{m}^{\prime}(x, y)=P^{-1}(x, y) P^{-1}(q x, r y) \cdots P^{-1}\left(q^{m-1} x, r^{m-1} y\right) T^{\prime}\left(q^{m} x, r^{m} y\right)$

Each element of $\boldsymbol{\Pi}_{m}^{\prime}(x, y)$ converges, for $k$ sufficiently large, to a definite limit function $v_{i j}(x, y)$, independent of $k$, as $m$ becomes infinite. This function is analytic throughout the finite $x$-and $y$-planes with the following exceptions: $x=0, y=0$, and places which are poles of an element of one of the matrices $P^{-1}(x, y), P^{-1}(q x, r y), \ldots$. For $x$ and $y$ outside certain associated circles about the origins in their respective planes and $x, y \neq \infty, v_{i j}(x, y)$ is identical with the corresponding element $s_{i j}^{\prime}(x, y)$ of $S^{\prime}(x, y)$.

The proof of this theorem runs entirely parallel to that of Theorem I, A; we shall omit the details and pass on to the statement of the corresponding pair of theorems which hold in

$$
\text { The Case of }|q|,|r|<1 \text {. }
$$

Here the two symbolic solutions of the equation (5), $P(x / q, y / r)$ - $P\left(x / q^{2}, y / r^{2}\right) \cdots$ and $P^{-1}(x, y) P^{-1}(q x, r y) \cdots$, interchange the rôles which they played in the discussion of the preceding case. The theorems follow.

Theorem I, C. Form the product of matrices

$$
\boldsymbol{\Pi}_{m}(x, y)=P^{-1}(x, y) P^{-1}(q x, r y) \cdots P^{-1}\left(q^{m-1} x, r^{m-1} y\right) T\left(q^{m} x, r^{m} y\right)
$$

Each element of $\boldsymbol{\Pi}_{\boldsymbol{m}}(x, y)$ converges, for $k$ sufficiently large, to a definite limit function $u_{i j}(x, y)$, independent of $k$, as $m$ becomes infinite. This function is analytic throughout the finite $x$ - and $y$-planes except perhaps at $x=0$, and except at places which are poles of an element of one of the matrices $P^{-1}(x, y), P^{-1}(q x, r y), \cdots$ For $x$ and $y$ inside certain associated circles $r_{1}, r_{2}$ about the origins in their respective planes and 
$x \neq 0, u_{i j}(x, y)$ is identical with the corresponding element $s_{i j}(x, y)$ of $S(x, y)$.

Theorem I, D. Form the product of matrices

$$
P_{m}^{\prime}(x, y)=P\left(\frac{x}{q}, \frac{y}{r}\right) P\left(\frac{x}{q^{2}}, \frac{y}{r^{2}}\right) \cdots P\left(\frac{x}{q^{m}}, \frac{y}{r^{m}}\right) T^{\prime}\left(\frac{x}{q^{m}}, \frac{y}{r^{m}}\right) .
$$

Each element of $P_{m}^{\prime}(x, y)$ converges, for $k$ sufficiently large, to a definite limit function $v_{i j}(x, y)$, independent of $k$, as $m$ becomes infinite. This function is analytic throughout the finite $x$ - and y-planes except at $x=0$ and $y=0$, and in this region is identical with the corresponding element $s_{i j}^{\prime}(x, y)$ of $S^{\prime}(x, y)$.

Of course the $u_{i j}(x, y)$ and $v_{i j}(x, y)$ of this case are entirely distinct from the solutions of the preceding case for which the same notation was used.

$$
\text { The Cases of }|q|>1,|r|<1 \text { and }|q|<1,|r|>1 \text {. }
$$

It is in the discussion of these cases that the matrices $S^{\prime \prime}(x, y)$ and $S^{\prime \prime \prime}(x, y)$ are of importance. We define a matrix $T^{\prime \prime}(x, y)\left[T^{\prime \prime \prime}(x, y)\right]$ by replacing the series there by power series in $x$ and $1 / y[1 / x$ and $y]$ which are known to converge in the vicinity of the place $(0, \infty)[(\infty, 0)]$ and which agree precisely with the series in $S^{\prime \prime}(x, y)\left[S^{\prime \prime \prime}(x, y)\right]$ up to and including terms of degree $k-1$ in $x$ and $1 / y$ [1/x and $y]$. Proceeding in entirely the same way as before we can prove, in each of the two cases, two theorems analogous to those above. We shall not pause to state the theorems here; it will suffice to remark that in both cases the series in the matrix $S^{\prime \prime}(x, y)\left[S^{\prime \prime \prime}(x, y)\right]$ converge for $x$ and $y$ respectively inside [outside] and outside [inside] certain associated circles $r_{1}, R_{2}\left[R_{1}, r_{2}\right]$ about the origins in their respective planes, and that by means of the equation (5) itself, analytic or meromorphic solutions are defined throughout a more extended region.

In the existence theorems for each of the four cases, use is made of but two of the four formal matrix solutions; for example, if $|q|$ and $|r|$ are both greater than unity, we have used only $S(x, y)$ and $S^{\prime}(x, y)$. It would be of interest to know just what is the significance of the two remaining formal solutions.

3. THE LINEAR EQUATION OF THE FIRST ORDER WITH POLYNOMIAL COEFFICIENT FOR THE CASE IN WHICH A CHARACTERISTIC EQUATION HAS THE ROOT ZERO

It will be recalled that in $\S 1$ one of the restrictions which it was found necessary to make in order to obtain the formal solutions (6), (7), 
(8), and (9) is that the roots of each characteristic equation be all different from zero. An important particular case is that in which the system (4) consists of a single equation (i. e., when $n=1$ ):

$$
\begin{aligned}
g(q x, r y) & =p(x, y) g(x, y), \\
p(x, y) & =p+p_{10} x+p_{01} y+\cdots+p_{\mu \nu} x^{\mu} y^{\nu} .
\end{aligned}
$$

In this case if $p=0$, the one root of the characteristic equation for $(0,0)$ vanishes and no formal series solutions of the type (6) can be found. Then if $|q|$ and $|r|$ are both $>$ or $<1$, one of the two solutions that are usually obtainable is lacking. We desire to show, however, that a solution can in general be found to take its place. We shall restrict our discusssion entirely to this case, although the method introduced is of rather general application. It can be used directly if $|q|$ and $|r|$ are both $>$ or $<1$ and a solution is missing because $p_{\mu \nu}=0$, and with slight modification if $|q|$ is $>1$ and $|r|$ is $<1$ or $|q|$ is $<1$ and $|r|$ is $>1$ and a solution is missing because $p_{0 \nu}=0$ or $p_{\mu 0}=0$. It may also be possible at times to use the method effectively when $n$ is $>1$ and one (or more) of the roots of a characteristic equation vanishes.

We shall accordingly assume $|q|,|r|>1$ or $|q|,|r|<1$ and proceed to obtain a solution for the equation (14) when $p=0$. If $p(x, y)$ can be factored into $x^{x} y^{\lambda}$ (where $x$ and $\lambda$ are positive integers or zero, but not both zero) multiplied by a polynomial $p^{\prime}(x, y)$ whose constant term is not zero, then a solution of (14) can be obtained by solving the two equations

$$
g_{1}(q x, r y)=x^{x} y^{\lambda} g_{1}(x, y), \quad g_{\mathbf{2}}(q x, r y)=p^{\prime}(x, y) g_{\mathbf{2}}(x, y),
$$

and multiplying the solutions together. The first of these equations is satisfied by

$$
g_{1}(x, y)=q^{\chi\left(t^{*}-t\right) / 2} r_{(\tau)-\tau) / 2}, \text { where } t=\frac{\log x}{\log q}, \tau=\frac{\log y}{\log r} .
$$

As for the second equation, that is solvable at once by the methods of $\S \S 1,2$.

A geometrical interpretation of what it means for $p(x, y)$ to be factorable into $x^{x} y^{\lambda} p^{\prime}(x, y)$ is worthy of notice. The terms of the polynomial $p(x, y)$ are of the form $x^{m} y^{n}$; let the point corresponding to each term be plotted in the $m, n$ plane. Clearly each such point will lie in the first quadrant or upon the positive $m$ - or $n$-axis, and a necessary and sufficient 
condition that $p(x, y)$ be so factorable is that there be one point of this set in the $m, n$ plane such that if there be drawn through it parallels to the axes, every other point of the set will lie above or upon the parallel to the $m$-axis and to the right or upon the parallel to the $n$-axis.

When $p(x, y)$ cannot thus be factored, our method of solving equation (14) will be to effect upon the variables $x$ and $y$ a transformation which will leave the given equation invariant in form but will change $p(x, y)$ into a polynomial $\bar{p}(\bar{x}, \bar{y})$ which can be factored in the desired manner. We propose to show that

$$
\begin{aligned}
& x=\bar{x}^{\alpha} \bar{y}^{\beta}, \\
& y=\bar{x}^{\gamma} \bar{y}^{\delta},
\end{aligned} \quad J=\alpha \delta-\beta \gamma \neq 0,
$$

where each of the exponents is a suitably determined positive integer or zero, is such a transformation. It is readily seen that under this transformation the equation (14) becomes

$$
\bar{g}(\bar{q} \bar{x}, \bar{r} \bar{y})=\bar{p}(\bar{x}, \bar{y}) \bar{g}(\bar{x}, \bar{y}),
$$

in which

$$
\bar{q}=\left(\frac{q^{\delta}}{r^{\beta}}\right)^{1 / J}, \quad \bar{r}=\left(\frac{r^{\alpha}}{q^{\gamma}}\right)^{1 / J}
$$

Hence it is our purpose to prove the following

Theorem. The quantities $\alpha, \beta, \gamma, \delta$ in (15), each of which is a positive integer or zero, can in general be so chosen that

(a) $\bar{p}(\bar{x}, \bar{y})$ will be factorable into $\bar{x}^{\bar{x}} \bar{y}^{\bar{\lambda}} \bar{p}^{\prime}(\bar{x}, \bar{y})$, where $\bar{p}^{\prime}(\bar{x}, \bar{y})$ has a constant term different from zero, and

(b) $|\bar{q}|$ and $|\bar{r}|$ will be both greater or both less than unity.

Proof. The effect of the transformation (15) on the polynomial $p(x, y)$ may best be observed in the $m, n$ plane. By (15) the term $x^{m} y^{n}$ goes into the term

$$
\bar{x}^{\bar{m}} \bar{y}^{\bar{n}}=\bar{x}^{\alpha m+\gamma n} \bar{y}^{\beta m+\delta n} .
$$

Thus the transformation of the $m, n$ plane which corresponds to (15) is

$$
\begin{aligned}
& \bar{m}=\alpha m+\gamma n, \\
& \bar{n}=\beta m+\delta n, \quad J=\alpha \delta-\beta \gamma \neq 0 .
\end{aligned}
$$


We shall think of this as a transformation of the axes, leaving the points of the plane fixed. Then the equations, referred to the $m, n$ axes, of the $\bar{m}, \bar{n}$ axes are respectively

$$
n=-\frac{\beta}{\delta} m, \quad n=-\frac{\alpha}{\gamma} m .
$$

These lines lie in the second and fourth quadrants, or at most, one of them may coincide with the $m$ - or the $n$-axis. Furthermore, since for $m$ and $n$ positive $\bar{m}$ and $\bar{n}$ are also positive, it follows that the angle $\theta$, less than $\pi$, between the positive $\bar{m}$ - and $\bar{n}$-axes must include as a part of itself the angle between the positive $m$ - and $n$-axes. By a proper choice of the exponents in (15) the $\bar{m}$ - and $\bar{n}$-axes can be put into any desired position of this type so long as the slopes of the $\bar{m}, \bar{n}$ axes are rational (or in particular, infinite).

Let us now consider again our set of points in the $m, n$ plane corresponding to terms of $p(x, y)$. A convex polygon can be drawn such that each vertex is a point of the set and every point of the set lies within or on the boundary of the polygon; let this polygon be constructed. Clearly it will have a vertex whose $m$-coördinate is less than that of any other vertex, or of any other but one. Call this point $A$; in case there are two such points, call the one with the larger $n$-coördinate $A$. Starting from this point proceed around the polygon in a counter-clockwise direction, lettering the successive vertices $B, C, \ldots$ until a vertex is reached whose $n$-coordinate is less than that of any other, or of any

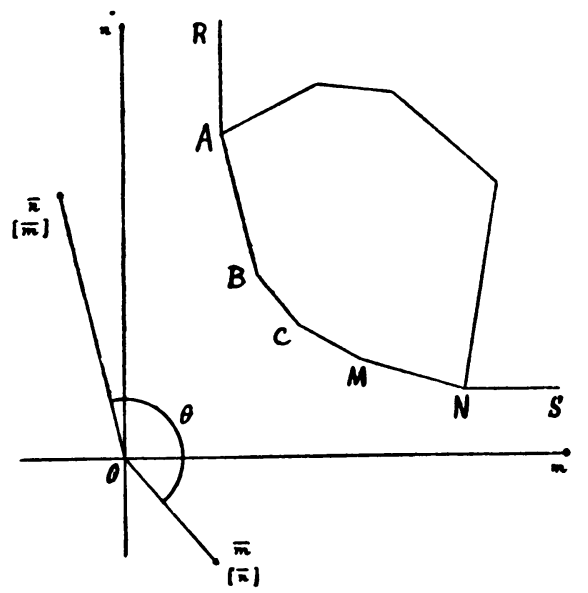
other except one. Let this last vertex be called $N$; or, if there are two such points, call the second one met (the one with the larger $m$-coordinate) $N$.

Now the slopes of all the lines $A B, B C, \ldots$ are rational and negative, or in particular infinite or zero. If the slope of $A B$ is not infinite, draw through $A$ a parallel $A R$ to the $n$-axis; if the slope of $M N$ is not zero, draw through $N$ a parallel $N S$ to the $m$-axis. Then it is possible given any vertex of the set $A, B, \ldots N$ (exclusive of $A$ if $A B$ has an infinite slope and exclusive of $N$ if $M N$ has a zero slope), say $B$ - to choose $\alpha, \beta, \gamma, \delta$ so that the $\bar{n}$ - and $\bar{m}$-axes [the $\bar{m}$ - and $\bar{n}$-axes] will 
be parallel respectively to $A R$ and $A B$, or to $B A$ and $B C$, or .., or to $N M$ and $N S$. Any of these choices will yield a transformation (15) which will take $p(x, y)$ into a polynomial of the desired factorable type.

Secondly consider the condition (b). By hypothesis we have $|q|,|r|>1$ or $|q|,|r|<1$, and it is necessary that $J$ be positive or negative. Let us assume $|q|,|r|>1$ and try to determine $\alpha, \beta, \gamma, \delta$ so that $J$ will be $>0$ and condition $(b)$, as well as condition $(a)$, will be satisfied. It is readily seen that $|\bar{q}|$ and $|\bar{r}|$ will both be greater [less] than unity if and only if

$$
\frac{\alpha}{\gamma}>\frac{\log |q|}{\log |r|}>\frac{\beta}{\delta} \quad\left[\frac{\beta}{\delta}>\frac{\log |q|}{\log |r|}>\frac{\alpha}{\gamma}\right] .
$$

The second of these possibilities, however, necessitates $J$ being $<0$. Hence if $|q|$ and $|r|$ are $>1$ and we are to use a transformation (15) in which $J$ is $>0$, a necessary and sufficient condition that the transformation satisfy the requirement $(b)$ is that $\alpha, \beta, \gamma, \delta$ be determined to satisfy the inequality

$$
\frac{\alpha}{\gamma}>\frac{\log |q|}{\log |r|}>\frac{\beta}{\delta} .
$$

But $\log |q| \div \log |r|$ is a positive constant. Its negative therefore lies, in general, between the values of the slopes of two consecutive lines of the set $R A, A B, B C, \ldots, M N, N S$; in particular, its negative may be equal to the slope of one of the lines $A B, B C, \ldots, M N$ ( $A B$ excluded if its slope is infinite and $M N$ if its slope is zero). In the general case, if $\alpha, \beta, \gamma, \delta$ be so chosen as to make the $\bar{n}$ - and $\bar{m}$-axes parallel respectively to the two lines of the set $R A, \ldots, N S$ between the values of whose slopes $-\log |q| \div \log |r|$ lies, then the condition $(b)$ as well as the condition $(a)$ will be satisfied. In the particular case a solution cannot be obtained by this method.

Several observations should be made at this point. First, by determining $\alpha, \beta, \gamma, \delta$ so that the rôles of the $\bar{m}$-and $\bar{n}$-axes in the preceding paragraph are interchanged, we obtain a transformation (15) satisfying $(a)$ and $(b)$ in which $J$ is negative and $|\bar{q}|$ and $|\bar{r}|$ are both less than unity. Secondly, in the case of $|q|,|r|<1$ a wholly similar discussion may be given. Thirdly, it is clear that in either case we can make $|\bar{q}|,|\bar{r}|>1$, so that the words "or both less" may be stricken from part $(b)$ in the statement of the theorem. 
A solution for the equation (16) can be found by solving the two equations

$$
\bar{g}_{1}(\bar{q} \bar{x}, \bar{r} \bar{y})=\bar{x}^{\bar{x}} \bar{y}^{\bar{\lambda}} \bar{g}_{1}(\bar{x}, \bar{y}), \quad \bar{g}_{2}(\bar{q} \bar{x}, \bar{r} \bar{y})=\bar{p}^{\prime}(\bar{x}, \bar{y}) \bar{g}_{2}(\bar{x}, \bar{y})
$$

and multiplying the solutions together; such a solution will be analytic throughout the finite $\bar{x}$ - and $\bar{y}$-planes except at $\bar{x}=0$ and $\bar{y}=0$. Transforming this solution into terms of $x$ and $y$ by the inverse of transformation (15) we obtain a solution of (14) which is analytic throughout the finite $x$ - and $y$-planes away from $x=0$ and $y=0$.

\section{THE CASE of $|q|=|r|=1$}

Theorem. When $|q|=|r|=1$ the equation of the class $\left(B^{\prime}\right)$ in general admits no analytic solution that is not identically zero.

We distinguish two sub-cases: (i) when at least one of the constants $q, r$ is not an $n$th root of unity for any positive integer $n$; (ii) when both $q$ and $r$ are roots of unity. The proofs will not be given here, for in each case a demonstration can be effected by the methods used by Carmichael* in proving the corresponding theorem for the linear ordinary $q$-difference equation; no very essential modification of Carmichael's work is needed beyond the introduction of a second independent variable.t

* Loc. cit., pp. 165-168.

† One point alone deserves special mention. In constructing a proof in subcase (i) it becomes necessary to show that, any positive integer $M$ being given and any $x$ and $y$ being assigned, an integer $n$ can be found which is $>M$ and such that the place $\left(q^{n} x, r^{n} y\right)$ is as near as you please to the place $(x, y)$. This problem is equivalent to that of finding an $n>M$ such that we shall have

$$
\left|q^{n}-1\right|<\varepsilon \quad \text { and } \quad\left|r^{n}-1\right|<\varepsilon,
$$

$\varepsilon(>0)$ being preassigned and arbitrarily small. If we set $\alpha=\arg q$ and $\beta=\arg r$, this reduces to determining integers $n(>M), m$, and $p$ to satisfy at the same time the two inequalities

$$
|n \alpha-m \cdot 2 \pi|<\varepsilon^{\prime} \quad \text { and } \quad|n \beta-p \cdot 2 \pi|<\varepsilon^{\prime} .
$$

That such a determination is possible follows immediately from a generalized form of Theorem III, pp. 7-8, of Minkowski's Diophantische Approximationen, Leipzig, Teubner, 1907. If in Minkowski's proof (p. 7) we allow his variable $z$ to take on the values $0, M, 2 M, \ldots, t^{2} M$, where $M$ is an arbitrarily assigned positive integer, the rest of the proof goes through without change, and we obtain a theoren which may be stated as follows (for $M=1$ it is Minkowski's): Given two arbitrary real numbers $a$ and $b$ and any two positive integers $M$ and $t$; there can always be found at least one integer $n, M \leqq n \leqq t^{2} M$, and two other integers $m$ and $p$ such that the following inequalities hold simultaneously:

$$
|n a-m|<\frac{1}{t}, \quad|n b-p|<\frac{1}{t} .
$$




\section{The PERIODIC FUNCTIONS}

The discussion of this section will be confined to the case of $|q|,|r|>1$; each of the other cases covered by the existence theorems of $\S 2$ may be treated in the same manner.

It has been shown in $\S 2$ that, under suitable restrictions, the matrix equation (5) admits of two matrix solutions, which may here be denoted for convenience by $S_{00}(x, y)$ and $S_{\infty \infty}(x, y)$ respectively. These solutions have been found to possess the following properties. $S_{00}(x, y)$ is analytic when $x(\neq 0)$ is inside an arbitrarily large circle $R_{1}$ about the origin in the $x$-plane and $y$ is inside an arbitrarily large circle $R_{2}$ about the origin in the $y$-plane, and is identically equal to $S(x, y)$. In a similar manner $S_{\infty \infty}(x, y)$ is analytic save for poles when $x(\neq \infty)$ and $y(\neq \infty)$ are respectively outside arbitrarily small circles $r_{1}, r_{2}$ about the origins in their respective planes. If $(x, y)$ lies within the region of convergence of the formal series solutions (7), we have $S_{\infty \infty}(x, y) \equiv S^{\prime}(x, y)$; if $(x, y)$ lies outside that region, $S_{\infty \infty}(x, y)$ is given by

$$
P^{-1}(x, y) P^{-1}(q x, r y) \cdots P^{-1}\left(q^{s-1} x, r^{s-1} y\right) S^{\prime}\left(q^{s} x, r^{s} y\right)
$$

where $s$ is a suitable positive integer.

We now define a matrix $A(x, y)$ by the equation

$$
S_{00}(x, y)=S_{\infty \infty}(x, y) A(x, y)
$$

from which it is clear that $A(x, y)$ is a matrix of multiplicatively periodic functions such that

$$
A(q x, r y)=A(x, y) .
$$

The elements of $A(x, y)$ are thus analytic, except perhaps for poles, in the region consisting of the $x$-plane between the circles $r_{1}$ and $R_{1}$ and of the $y$-plane between the circles $r_{2}$ and $R_{2}$; it will first be proved that no such poles can occur.

We have $A(x, y)=S_{\infty \infty}^{-1}(x, y) S_{00}(x, y)$. The elements of $S_{\infty \infty}^{-1}(x, y)$ may have singularities at the poles of elements of $S_{\infty \infty}(x, y)$ and at the zeros of the determinant $\left|S_{\infty}(x, y)\right|$. But outside of certain definite associated circles $\bar{R}_{1}, \bar{R}_{2}$ the elements of $S_{\infty \infty}(x, y)$, so long as neither $x$ nor $y$ is actually infinite, have no poles, and outside certain other definite associated circles $\overline{\bar{R}}_{1}, \overline{\bar{R}}_{2}$ the determinant $\left|S_{\infty}(x, y)\right|$, so long as neither 
$x$ nor $y$ is actually infinite, does not vanish.* Now let any place $\left(x_{1}, y_{1}\right)$ be chosen such that $x_{1}$ lies between $r_{1}$ and $R_{1}$ and $y_{1}$ lies between $r_{2}$ and $R_{2}$. We have

$$
A\left(x_{1}, y_{1}\right)=A\left(q x_{1}, r y_{1}\right)=\cdots=A\left(q^{s} x_{1}, r^{s} y_{1}\right),
$$

and $s$ can be taken so large that $q^{s} x_{1}$ will lie outside the larger of the circles $\bar{R}_{1}, \overline{\bar{R}}_{1}$ and yet inside $R_{1}$, and $r^{s} y_{1}$ will lie outside the larger of the circles $\bar{R}_{2}, \overline{\bar{R}}_{2}$ but inside $R_{2}$. Hence $A(x, y)$ must be analytic at $\left(q^{s} x_{1}, r^{s} y_{1}\right)$ and therefore at $\left(x_{1}, y_{1}\right)$. From this we conclude

TheOREM II, AB. The elements of the matrix $A(x, y)$ defined by (17) are functions possessing the multiplicative period system $(q, r)$; these functions are analytic in the region consisting of the x-plane between circles $r_{1}$ and $R_{1}$ about the origin and of the y-plane between circles $r_{\mathbf{2}}$ and $R_{\mathbf{2}}$ about the origin, where $r_{1}$ and $r_{2}$ are arbitrarily small and $R_{1}$ and $R_{2}$ are arbitrarily large.

We shall find it worth while to examine these periodic functions a little more in detail. In order that they may be single-valued functions of position it will be convenient to think of each of the variables $x$ and $y$ as free to range over an infinitely many-sheeted Riemann surface of the type used for the logarithm, with branch points of infinite order at the origin and at infinity. Let us then make the transformation

$$
x=q^{t}, \quad y=r^{\tau},
$$

which maps the Riemann surfaces for $x$ and $y$ respectively in a one-to-one and conformal manner on the $t$-plane and $\tau$-plane, and define

$$
A(x, y)=B(t, \tau)
$$

$B(t, \tau)$ is thus a matrix of functions single-valued and analytic over the region made up of the portions of the $t$-plane and $\tau$-plane within arbitrarily large circles about the respective origins. Moreover the elements of $B(t, \tau)$ have additive, rather than multiplicative, period systems.

Let $x$ now be made to traverse a positive circuit about the origin in its own plane ( $y$ remaining fixed). $S_{00}(x, y)$ becomes $S_{00}(x, y) K$, where $K$ stands for the matrix $\left(e^{2 \pi \rho_{j} V-1} \delta_{i j}\right), \delta_{i j}$ being as before the Kronecker $\delta$.

* Cf. inequalities (10). 
At the same time $S_{\infty \infty}(x, y)$ changes to

$$
(-1)^{\mu} e^{2 \pi V \overline{-1} \mu t} e^{-2 \pi^{2} \mu / \log q} S_{\infty \infty}(x, y) L
$$

where $L$ is the matrix $\left(e^{2 \pi \rho_{j}^{\prime} \sqrt{-1}} \delta_{i j}\right)$. Thus when $x$ has made a positive circuit of the origin, $A(x, y)$ is replaced by

$$
(-1)^{\mu} e^{-2 \pi \sqrt{-1} \mu t} e^{2 \pi^{2} \mu / \log q} L^{-1} A(x, y) K
$$

If, then, $a_{i j}(x, y)$ is the element in the $i$ th row and $j$ th column of $A(x, y)$, the passage of $x$ around such a circuit changes $a_{i j}(x, y)$ to

$$
(-1)^{\mu} e^{-2 \pi \sqrt{-1} \mu t} e^{2 \pi^{2} \mu / \log q} e^{2 \pi V-1\left(-\rho_{i}^{\prime}+\rho_{j}\right)} a_{i j}(x, y)
$$

Similarly if $y$ be caused to traverse a circuit about the origin in the positive sense ( $x$ remaining fixed), $a_{i j}(x, y)$ is replaced by

$$
(-1)^{\nu} e^{-2 \pi \sqrt{-1} \nu \tau} e^{2 \pi^{2} \nu / \log r} a_{i j}(x, y)
$$

It follows that if $b_{i j}(t, \tau)$ denotes the element in the $i$ th row and $j$ th column of $B(t, \tau)$, then

$$
b_{i j}(t+1, \tau+1)=b_{i j}(t, \tau)
$$

$$
\begin{aligned}
& b_{i j}\left(t+\frac{2 \pi \sqrt{-1}}{\log q}, \tau\right)=\left(-1_{i}\right)^{\mu} e^{-2 \pi \sqrt{-1}\left(\mu t+\rho_{i}^{\prime}-\rho_{j}\right)+2 \pi^{2} \mu / \log q} b_{i j}(t, \tau) \\
& b_{i j}\left(t, \tau+\frac{2 \pi \sqrt{-1}}{\log r}\right)=(-1)^{\nu} e^{-2 \pi \sqrt{-1} \nu \tau+2 \pi^{2} \nu / \log r} b_{i j}(t, \tau)
\end{aligned}
$$

Thus the elements of the matrix $B(t, \tau)$ are seen to be triply periodic, or perhaps one might better say "triply quasi-periodic." As such these functions are a special case of the functions treated by Cousin* in a memoir in which he discusses, with only slight restrictions, the properties of the general meromorphic function of two complex variables whose zeros admit

* Sur les fonctions triplement périodiques de deux variables, Acta Mathematica, vol. 33 (1910), pp. 105-232. 
three systems of periods.* These restrictions are in general satisfied by the functions $b_{i j}(t, \tau)$. The functions $b_{i j}(t, \tau)$ are a more restricted type than those dealt with by Cousin in that they are entire, rather than meromorphic frnctions and in that the exponents of $e$ in the equations (18) are linear functions of $t$ and $\tau$ rather than the wholly general entire functions which appear in the corresponding equations of Cousin. The Cousin paper also shows how a triply periodic function can be expressed in explicit form in terms of series of the type

$$
\sum_{n=-\infty}^{+\infty} \boldsymbol{\Phi}_{n}(t) e^{n \tau}
$$

in which $\Phi_{n}(t)$ is essentially an exponential function of $t$ multiplied by a product of $\theta$-functions of $t-a_{i}^{(n)}\left(a_{i}^{(n)}\right.$ being a constant $\left.(i=1,2, \ldots, k)\right)$. Hence the functions $b_{i j}(t, \tau)$ are expressible in such a manner as this, which is remotely analogous to the explicit form found by Birkhofft for the periodic functions that arise in the theory of the linear ordinary $q$-difference equation.

\section{A MORE GENERAL PROBLEM; AN INVERSE THEOREM}

In the four matrices of formal series solutions as they were found in $\S 1$, there seems to be a lack of symmetry. This lack, however, is only apparent, for in defining our characteristic equations we might equally well have proceeded as follows. Among all the polynomials $p_{i j}(x, y)$ there will be one (or more) which contains $x[y]$ to a maximum power; let that power be $\mu[\nu]$; similarly there will be one (or more) which contains $x[y]$ to a minimum power; let that power be $x[\lambda]$. Then the characteristic equations are

$$
\begin{array}{ll}
\text { for }(0,0), & \left|p_{i j \times \lambda}-\delta_{i j} \sigma\right|=0 ; \\
\text { for }(\infty, \infty), & \left|p_{i j \mu \nu}-\delta_{i j} \sigma^{\prime}\right|=0 ; \\
\text { for }(0, \infty), & \left|p_{i j \chi \nu}-\delta_{i j} \sigma^{\prime \prime}\right|=0 ; \\
\text { for }(\infty, 0), & \left|p_{i j \mu \lambda}-\delta_{i j} \sigma^{\prime \prime \prime}\right|=0 ;
\end{array}
$$

\footnotetext{
* The zeros of $f(t, \tau)$ are said to admit the system of periods $(a, b)$ if $f(t+a, \tau+b)$ $=e^{g(t, \tau)} f(t, \tau)$, where $g(t, \tau)$ is an entire function.

†The generalized Rienann problem for..., Proceedings of the American Academy of Arts and Sciences, vol. 49 (1913), pp. 561-564.
} 
and the formal matrix solutions are found to be

$$
\begin{aligned}
& S(x, y)=\left(q^{\chi\left(t^{2}-t\right) / 2} r^{\lambda\left(\tau^{2}-\tau\right) / 2} x^{\rho_{j}}\left[s_{i j}+s_{i j 10} x+s_{i j 01} y+\cdots\right]\right), \\
& S^{\prime}(x, y)=\left(q^{\mu\left(t^{2}-t\right) / 2} r^{\nvdash\left(\tau^{2}-\tau\right) / 2} x^{\rho_{j}^{\prime}}\left[s_{i j}^{\prime}+\frac{s_{i j 10}^{\prime}}{x}+\frac{s_{i j 01}^{\prime}}{y}+\cdots\right]\right), \\
& S^{\prime \prime}(x, y)=\left(q^{x\left(t^{\natural}-t\right) / 2} r^{\nu\left(\tau^{2}-\tau\right) / 2} x^{\rho_{j}^{\prime \prime}}\left[s_{i j}^{\prime \prime}+s_{i j 10}^{\prime \prime} x+\frac{s_{i j 01}^{\prime \prime}}{y}+\cdots\right]\right), \\
& S^{\prime \prime \prime}(x, y)=\left(q^{\mu\left(l^{2}-t\right) / 2} r^{\chi\left(\tau^{2}-\tau\right) / 2} x^{\rho_{j}^{\prime \prime \prime}}\left[s_{i j}^{\prime \prime \prime}+\frac{s_{i j 0}^{\prime \prime \prime}}{x}+s_{i j 01}^{\prime \prime \prime} y+\cdots\right]\right) .
\end{aligned}
$$

In this form there is evidently complete symmetry, and we note that when $x=\lambda=0$ the matrices reduce to those of $\S 1$.

It immediately becomes clear, after the preceding remark, that the polynomials $p_{i j}(x, y)$ may just as well be taken as polynomials in $x, 1 / x$, $y$, and $1 / y$. In that event the formal work of the foregoing paragraph is unchanged, but $x$ and $\lambda$ are negative (i. e., if terms in $1 / x$ and $1 / y$ actually occur). Furthermore it should be observed that in the cases of $|q|,|r|>1$ and of $|q|,|r|<1$, existence theorems can be proved almost entirely as above if the elements of the matrix are any functions whatever which are expressible in each of the following forms, the first valid in the vicinity of $(0,0)$ and the second valid in the vicinity of $(\infty, \infty)$ :

$$
x^{x} y^{\lambda} \text { [convergent power series in } x \text { and } y \text { ], }
$$

$$
x^{\mu} y^{\nu}\left[\text { convergent power series in } \frac{1}{x} \text { and } \frac{1}{y}\right]
$$

(provided, of course, the restrictive conditions imposed in $\S 1$ are satisfied); the only difference in the results is that the solutions obtained may be valid only in definitely limited regions about $(0,0)$ and $(\infty, \infty)$ respectively. It is clear that a similar remark applies in the two remaining cases, $|q|>1,|r|<1$ and $|q|<1,|r|>1$.

We are now in a position to prove a theorem which is in part an inverse of some of the results that have been found in this and preceding sections. 
Theorem III, ABCD. Let $G(x, y)$ and $H(x, y)$ be two matrices whose elements, after multiplication by $q^{-x(\boldsymbol{r}-t) / 2} r^{-\lambda\left(\tau^{s}-\tau\right) / 2} x^{-\rho_{s}}$ in the case of $G(x, y)$ and by $q^{-\mu\left(t^{-}-t\right) / 2} r^{-\nu\left(\tau^{*}-\tau\right) / 2} x^{-\rho_{j}^{\prime}}$ in the case of $H(x, y)$, are singlevalued functions analytic save for poles over the extended $x$ - and $y$-planes with the exception of the places $(0,0)$ and $(\infty, \infty)$ and such that

$$
\begin{aligned}
& \lim _{\substack{x=0 \\
y=0}} g_{i j}(x, y) q^{-x\left(t^{3}-t\right) / 2} r^{-\lambda\left(\tau^{3}-\tau\right) / 2} x^{-\rho_{j}}=s_{i j}, \\
& \lim _{\substack{x=\infty \\
y=\infty}} h_{i j}(x, y) q^{-\mu\left(t^{\natural}-t\right) / 2} r^{-\nu\left(\tau^{s}-\tau\right) / 2} x^{-\rho_{j}^{\prime}}=s_{i j}^{\prime}, \quad\left(\begin{array}{l}
|,| r \mid>1 \text { or } \\
|q|,|r|<1
\end{array}\right)
\end{aligned}
$$

where the s's and @'s are constants subject to the conditions

$$
\begin{gathered}
\left|s_{i j}\right| \neq 0, \quad\left|s_{i j}^{\prime}\right| \neq 0 ; \\
\varrho_{i}-\varrho_{j} \neq \beta+\gamma \frac{\log r}{\log q}, \quad \varrho_{i}^{\prime}-\varrho_{j}^{\prime} \neq \beta+\gamma \frac{\log r}{\log q}
\end{gathered}
$$

for any $\beta$ and $\gamma$ that are positive or negative integers or zero $(i \neq j)$; and where $x, \lambda, \mu$ and $\nu$ are integers (or zero) and

$$
t=\frac{\log x}{\log q}, \quad \tau=\frac{\log y}{\log r} .
$$

Also let the two matrices be connected by the relation

$$
G(x, y)=H(x, y) A(x, y),
$$

in which $A(x, y)$ is a matrix of functions possessing the multiplicative period system $(q, r)$. Then $G(x, y)$ and $H(x, y)$ are solutions of a system (5) in which the elements of $P(x, y)$ are rational functions.

Proof. By hypothesis we have

$$
H^{-1}(q x, r y) G(q x, r y)=H^{-1}(x, y) G(x, y) .
$$

Let $P(x, y)$ denote the product of matrices

$$
G(q x, r y) G^{-1}(x, y)=H(q x, r y) H^{-1}(x, y)
$$


its elements are single-valued and analytic except for poles over the extended $x$ - and $y$-planes apart from the places $(0,0)$ and $(\infty, \infty)$.

Upon calculating the elements $\bar{g}_{i j}(x, y)\left[\bar{h}_{i j}(x, y)\right]$ of $G^{-1}(x, y)\left[H^{-1}(x, y)\right]$, it becomes clear, by virtue of our hypotheses, that

$$
\begin{aligned}
& \lim _{\substack{x=0 \\
y=0}} \bar{g}_{i j}(x, y) q^{x\left(t^{2}-t\right) / 2} r^{\lambda\left(\tau^{2}-\tau\right) / 2} x^{\rho_{i}}=\overline{s_{i j}} \\
& {\left[\lim _{\substack{x=\infty \\
y=\infty}} \bar{h}_{i j}(x, y) q^{\mu\left(\boldsymbol{t}^{\boldsymbol{2}}-t\right) / 2} r^{r\left(\tau^{\boldsymbol{*}}-\tau\right) / 2} x^{\rho_{i}^{\prime}}=\bar{s}_{i j}^{\prime}\right],}
\end{aligned}
$$

where $\bar{s}_{i j}\left[\bar{s}_{i j}^{\prime}\right]$ is the element in the $i$ th row and $j$ th column of the inverse matrix of $\left(s_{i j}\right)\left[\left(s_{i j}^{\prime}\right)\right]$. Hence, if $(x, y)$ is in the neighborhood of $(0,0)$, we have

$$
p_{i j}(x, y)=\sum_{\alpha=1}^{n} g_{i \alpha}(q x, r y) \bar{g}_{\alpha j}(x, y)=x^{x} y^{\lambda} \sum_{\alpha=1}^{n} q^{\rho_{\alpha}}\left(s_{i \alpha} \bar{s}_{\alpha j}+\eta_{i j \alpha}\right),
$$

where $\eta_{i j \alpha}(i, j, \alpha=1,2, \ldots, n)$ approaches zero as $(x, y)$ approaches $(0,0)$. Similarly, if $(x, y)$ is in the neighborhood of $(\infty, \infty)$, we have

$$
p_{i j}(x, y)=x^{\mu} y^{\nu} \sum_{\alpha=1}^{n} q^{\rho_{\alpha}^{\prime}}\left(s_{i \alpha}^{\prime} \bar{s}_{\alpha j}^{\prime}+\eta_{i j \alpha}^{\prime}\right)
$$

in which $\eta_{i j \alpha}^{\prime}(i, j, \alpha=1,2, \ldots, n)$ approaches zero as $(x, y)$ approaches $(\infty, \infty)$. It follows that the elements of $P(x, y)$ are analytic or have poles at $(0,0)$ and at $(\infty, \infty)$. Consequently they are analytic except for poles over the extended $x$ - and $y$-planes without exception, and are rational functions of $x$ and $y$.

We have, then, the two equations

$$
\begin{aligned}
& G(q x, r y)=P(x, y) G(x, y), \\
& H(q x, r y)=P(x, y) H(x, y) .
\end{aligned}
$$

From the preceding paragraph it follows that the elements of $P(x, y)$ have at $(0,0)$ and at $(\infty, \infty)$ the requisite forms (19). It remains to show that the roots of the characteristic equations for $(0,0)$ and for $(\infty, \infty)$ 
satisfy the conditions imposed in $\S 1$. To do this let us rewrite (20) in the form

$$
g_{i j}(q x, r y)=\sum_{u=1}^{n} p_{i u}(x, y) q_{u j}(x, y) \quad(i, j=1,2, \ldots, n) .
$$

For any particular $i$ this system provides $n$ linear non-homogeneous equations for the determination of the $n$ functions $p_{i u}(x, y)(u=1,2, \ldots, n)$. If they be solved for $p_{i u}(x, y)$ and $(x, y)$ be allowed to approach $(0,0)$, the coefficient in the leading term of the expansion for $p_{i u}(x, y)$ at $(0,0)$ being denoted by $p_{i u x \lambda}$, we have

$$
p_{i u x \lambda}=\frac{\left|s_{l m}^{(i u)}\right|}{\left|s_{l m}\right|}
$$

where $\left|s_{l m}^{(i u)}\right|$ stands for the determinant obtained from $\left|s_{l m}\right|$ by replacing the elements of the $u$ th row by $q^{\rho_{j}} s_{i j}(j=1,2, \ldots, n)$. (It is clearly possible thus to solve, because by hypothesis $\left|s_{l m}\right| \neq 0$.) The characteristic equation for $(0,0)$ is

$$
\left|p_{i u \times \lambda}-\delta_{i u} \sigma\right|=0
$$

upon examination its roots are seen to be $q^{\rho_{1}}, q^{\rho_{2}}, \ldots, q^{\rho_{n}}$. In the same manner we may show from (21) that, if $p_{i u \mu \nu}$ is the coefficient in the leading term of the expansion for $p_{i u}(x, y)$ at $(\infty, \infty)$, then we have

$$
p_{i u \mu \nu}=\frac{\left|s_{l m}^{\prime(i u)}\right|}{\left|s_{l m}^{\prime}\right|}
$$

and that the roots of the characteristic equation for $(\infty, \infty)$,

$$
\left|p_{i u \mu \nu}-\delta_{i u} \sigma^{\prime}\right|=0
$$

are $q^{\rho_{1}^{\prime}}, q^{\rho_{2}^{\prime}}, \ldots, q^{\rho_{n}^{\prime}}$. It then follows from our hypotheses that the matrix satisfies all the conditions that it has been found necessary, in our earlier work, to impose upon the matrix of coefficients in the system (5). This completes the proof of the theorem. 
If one of the two numbers $|q|$ and $|r|$ is greater and the other less than unity, an entirely similar theorem can be stated and proved.

In conclusion, we may call attention to the fact that a discussion of the equation $\left(B^{\prime}\right)$ such as has been given covers also the cases of equations $\left(A^{\prime}\right)$ and $\left(C^{\prime}\right)$ for certain types of coefficient functions. In the first place, subjecting equation (5) to the transformation $x=q^{t}, y=r^{\tau}$, we see at once that our discussion disposes of the case of a system of equations of the first order of the class $\left(A^{\prime}\right)$ when the coefficients are polynomials (or such other functions as have been noted above) in $q^{x}$ and $r^{y}$. Secondly, if in (5) we set $x=q^{t}, y=\tau\left[x=t, y=r^{\tau}\right]$, we find that our work has covered the case of a system of equations of the first order of the class $\left(C^{\prime}\right)$ with coefficients which are polynomials (or such other functions as we have noted above) in $q^{x}$ and $y$ [x and $\left.r^{y}\right]$.

Brown University,

Providence, R. I. 